SHAHRIAR AFANDIZADEH, Ph.D.

E-mail: zargari@iust.ac.ir

SEYED EBRAHIM ABDOLMANAFI, Ph.D. Candidate

(Corresponding author)

E-mail: abdolmanafi@iust.ac.ir

School of Civil Engineering

Iran University of Science and Technology

Narmak, Tehran, 1684613114, Iran
Traffic and Environment (Ecology)

Preliminary Communication

Submitted: Apr. 23, 2015

Accepted: Jan. 29, 2016

http://dx.doi.org/10.7307/ptt.v28i2.2022

\title{
CORDON PRICING CONSIDERING AIR POLLUTANTS EMISSION
}

\begin{abstract}
This paper considers the issue of air pollutants emission for the optimal and sustainable determination of cordon location, toll level, and price of park and ride (P\&R). Although air pollutants emission decreases within the cordon by the implementation of cordon pricing scheme, it may increase outside the cordon and the whole network. Hence, air pollutants emission may only transfer from inside of the cordon to its outside. Therefore, in this paper, a multi-objective bi-level optimization model is developed. A solution algorithm is also presented based on the second version of strength Pareto evolutionary algorithm (SPEA2). The results reveal that this multi-objective model can be a useful tool for the sustainable and optimal design of the cordon and P\&R scheme. In addition, cordon pricing is a multi-objective problem. Therefore, it is necessary to consider air pollutants emission. By choosing another non-dominated result in the solution space, air pollutants emission outside the cordon and the whole network can be reduced without a significant reduction in social welfare.
\end{abstract}

\section{KEY WORDS}

cordon location; toll level; park and ride; air pollutants emission; sustainable development; SPEA2 method;

\section{INTRODUCTION}

Traffic congestion has become one of the most severe social problems in modern societies. For years, adding additional capacity has been the solution for the rising level of congestion [1]. However, such an approach is subject to many spatial and financial constraints. Furthermore, providing more road space has been proven to be self-defeating in congested areas, because increased capacity will soon be occupied by induced travel demands [2, 3]. Thus, in order to alleviate roadway congestion, road congestion pricing has been introduced.

Congestion pricing was first suggested by investigating a sample of a congested road and expressing some ideas about externalities and optimal congested charges by Pigou (1920). The fundamental concept of pricing is so simple: prices should be higher under congestion conditions and lower at less congested times and locations in order to prevent excessive use [4].
Recently, road pricing issue has widely attracted the attention of economists and transportation researchers, due to growing prominence and changing nature of urban transportation problems faced by modern cities [5-7].

Road pricing theory is based on the fundamental economic principle of marginal cost pricing. It indicates that the users who use congested roads have to pay a toll which is equal to the difference between marginal social cost and marginal private cost in a way that social surplus increases [8]. The marginal cost pricing, unlike its full theoretical basis, is of little practical interest. Therefore, the second-best pricing method has attracted interest recently [4]. In the second-best pricing method, toll is only charged over a subset of links of the network. Four types of toll charging scheme in road network seem to be more popular: travel-distance based charging, travel-delay based charging, linkbased charging, and cordon-based charging [9].

Recently, in some countries, a cordon pricing scheme has been used, instead of pricing on separate individual links, in order to reduce traffic demand in central congested urban areas [10-13]. In the second-best pricing, simultaneous determination of toll locations and toll level on a network is practically important [14-16]. In addition, the effect of value of time (VOT) on the pricing problems has been investigated in some of the previous studies [17]. In the presence of heterogeneous users with different VOTs, various network equilibrium models have been developed either by assuming a discrete set of VOTs for several distinct user classes or by a continuously distributed VOT across the whole population [18-23]. Moreover, in other studies, equity issues and revenue redistribution in congestion pricing have been investigated [24-28]. Recently, different types of toll design including timetoll, distance-toll, and speed-based toll have been considered for cordon-based pricing scheme [29, 30].

In terms of environmental impacts, differential distribution of environmental risk on users and places has been examined in most studies [31]. Previous works have employed various spatial, analytical, and statistical techniques to examine the distribution and potential impacts of locally undesirable land uses 
$[32,33]$. In addition, a few studies have used GISbased proximity analysis to examine environmental effects of transportation [34]. Moreover, some investigations have considered the environmental justice implications of transportation plans and policies [35]. In fact, most of these studies have been only focused on the environmental impacts of transportation projects. However, the issue of air pollutants emission in designing the cordon charging scheme have not been investigated.

We believe that the cordon charging scheme is a multi-objective problem. One of the ignored objective functions in this problem is air pollutants emission. In addition to social welfare objective function, air pollutants emission is effective in the cordon pricing scheme. In other words, by an increase in social welfare, air pollutants emission may not decrease in the network. Rather, it may only transfer from the inside of the cordon to its outside. Therefore, it may increase outside the cordon and the whole network. The present paper considers air pollutants emission as an objective function in the cordon charging and P\&R scheme.

The rest of this paper is organized as follows. In Section 2, the problem of air pollutants emission is described by the implementation of cordon charging in an artificial network. In Section 3, a mathematical programming model is presented for the optimal and simultaneous determination of the cordon location, toll level, and price of P\&R. In Section 4, a solution algorithm is presented for solving the developed model based upon the second version of strength Pareto evolutionary algorithm (SPEA2). In addition, an innovative method based on geometric coordinate is proposed for dealing with the logical constraint of meta-heuristic algorithms. In Section 5, the developed model is applied to the Sioux Falls network, as a numerical example, and the results of the developed model are illustrated and discussed. Finally, summary and concluding remarks are provided in Section 6.

\section{PROBLEM OF AIR POLLUTION WITH CORDON PRICING}

To illustrate the issue of air pollutants emission, an artificial network with four nodes and four links is used as shown in Figure 1. The artificial network consists of two different OD pairs, from 1 to 4 and 2 to 4 . Travel demands from origin 1 to destination 4 and from origin 2 to destination 4 are considered 400 and 300, respectively. The length of each link is shown inside the parentheses. Travel cost functions are equal to:

$$
\begin{aligned}
& t_{1}\left(v_{1}\right)=2.5+\frac{v_{1}}{400} \\
& t_{2}\left(v_{2}\right)=1.0+\frac{v_{2}}{200} \\
& t_{3}\left(v_{3}\right)=1.0+\frac{v_{3}}{400} \\
& t_{4}\left(v_{4}\right)=0.5+\frac{v_{4}}{400}
\end{aligned}
$$

First, it is assumed that there is no cordon pricing scheme; so, all the links are toll-free (Case 1). Second, it is supposed that charging a toll is equal to 0.5 minute in link 4 (Case 2). Assuming the application of deterministic user equilibrium (DUE), traffic volume and average speed of traffic flow in the links can be estimated in two cases. Then, by considering the link length and equilibrium traffic volume, we can calculate the total air pollutants emission in each link and the network in two cases (Table 1).

Thus, the corresponding ratios of the air pollutants emission after and before implementation of cordon

\begin{tabular}{|c|c|c|c|c|c|c|}
\hline \multirow{2}{*}{ Link } & \multicolumn{2}{|c|}{ Traffic volume (vehicle) } & \multicolumn{2}{|c|}{ Speed $(\mathrm{km} / \mathrm{h})$} & \multicolumn{2}{|c|}{ Total air pollution (kg) } \\
\hline & Case1 & Case2 & Case1 & Case2 & Case1 & Case2 \\
\hline 1 & 275 & 325 & 60 & 56 & 4.61 & 5.61 \\
\hline 2 & 125 & 75 & 48 & 49 & 0.47 & 0.28 \\
\hline 3 & 300 & 300 & 50 & 50 & 1.25 & 1.25 \\
\hline 4 & 425 & 375 & 32 & 34 & 5.13 & 4.37 \\
\hline \multicolumn{5}{|c|}{ Network } & 11.46 & 11.50 \\
\hline
\end{tabular}
charging scheme in each link and the network are presented in Table 2.

Table 1 - Total air pollutants emission in two cases

Table 2 - Comparison of the results in two cases

\begin{tabular}{|c|c|c|c|c|c||}
\hline Link & 1 & 2 & 3 & 4 & Network \\
\hline \hline Ratios of air pollution & 1.22 & 0.59 & 1.00 & 0.85 & 1.004 \\
\hline
\end{tabular}




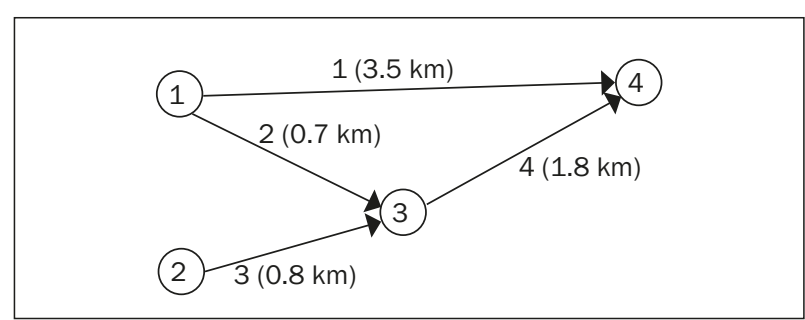

Figure 1 - A simple artificial network

The comparison of results of the air pollution emission in two cases, without and with cordon pricing shows that the implementation of cordon charging scheme may increase air pollutants emission in the whole network. In other words, although air pollutants emission decreases within the cordon (link 2), this policy may only shift air pollutants emission from inside of cordon to the outside.

\section{MODEL FORMULATION}

The issue of cordon pricing scheme is a transportation network optimization problem with user equilibrium constraints [36-38].

\subsection{The lower level of the developed model and its solution algorithm}

In fact, the lower level problem in cordon charging is user equilibrium. For solving the user equilibrium problem, the following assumptions are considered:

1) Travel demand is elastic.

2) There are three transportation modes in the network, namely private car, taxi, and bus.

3) P\&Rs exist at the cordon boundary.

Note that for solving the user equilibrium problem the elastic demand based upon an iterative diagonalization process is changed to the fixed demand and then solved; after each step, the convergence of the demand is examined. The steps of the solution algorithm for a lower level problem are as follows:

Step 0: Assuming initial travel time using Expressions 5 ,

$t_{a}^{C}=t_{a}^{0}, t_{a}^{T}=t_{a}^{C}=t_{a}^{0}, t_{a}^{B}=1.2 t_{a}^{C}=1.2 t_{a}^{0}$

where $t_{a}^{c}, t_{a}^{T}$, and $t_{a}^{B}$ are travel time of cars, taxis, and buses in link 'a', respectively. $t_{a}^{0}$ is free flow travel time in link ' $a$ '.

Step 1: Calculating the minimum travel costs of each mode between OD pair 'w' using Expressions 6 to 8 ,

$$
\begin{aligned}
& u_{w}^{C}=\min \left[\sum_{a \in A} \delta_{a, k}\left(t_{a}^{C}+\delta_{a} \tau_{a}\right)\right] \\
& u_{w}^{T}=\min \left[\sum_{a \in A} \delta_{a, k}\left(t_{a}^{T}\right)\right] \\
& u_{w}^{B}=\min \left[\sum_{a \in A} \delta_{a, k}\left(t_{a}^{B}\right)\right]
\end{aligned}
$$

where $u_{w}^{c}, u_{w}^{T}$, and $u_{w}^{B}$ are minimum travel costs of cars, taxis, and buses between OD pair $w \in W$, respectively. $\tau_{a}$ is toll level in link ' $a$ '. If link ' $a$ ' is tolled, $\delta_{a}$ is one; otherwise, it equals zero. If link 'a' belongs to path ' $k$ ' between origin ' $r$ ' and destination ' $s$ ', $\delta_{a, k}$ is one; otherwise, it is zero.

Step 2: Calculating the travel demand of modes between OD pair ' $w$ ' using Expression 9,

$$
d_{w}^{m}=Q_{w} \exp \left(-\gamma_{w} \mu_{w}\right) \frac{\exp \left(a_{m} u_{w}^{m}+b_{m}\right)}{\sum_{m=C, T, B} \exp \left(a_{m} u_{w}^{m}+b_{m}\right)}
$$

where $a_{m}$ and $b_{m}$ are constant coefficients that are calibrated by network data. $d_{w}^{m}$ is travel demand between OD pair $w \in W$ with mode ' $m$ '. $Q_{W}$ is initial total travel demand between OD pair ' $w$ '. $\gamma_{w}$ is demand elasticity coefficient between OD pair ' $w$ ' that is related to network condition. $\mu_{w}$ is minimum travel cost between OD pair ' $w$ '.

Step 3: Modifying car travel demand due to the existence of P\&Rs at the cordon boundary; some drivers may shift from private cars to taxis or buses. The modification procedure includes:

a) Identifying car travel demand whose destination is within the cordon;

b) Determining the nearest $P \& R$ to origin ' $r$ ' and destination 's' as a mid-point ' $p$ ';

c) Calculating the minimum travel costs of modes. Based upon the mid-point (P\&R 'p'), minimum travel costs of modes is calculated under three conditions (car only, car-taxi, and car-bus) using Expressions 10 to 13 ,

$$
\begin{aligned}
& u_{r p}^{c}=\min \left[\sum_{a \in A} \delta_{a, k}\left(t_{a}^{c}\right)\right] \\
& u_{p s}^{c}=\min \left[\sum_{a \in A} \delta_{a, k}\left(t_{a}^{c}+\tau_{a}\right)\right] \\
& u_{p s}^{T}=\min \left[\sum_{a \in A} \delta_{a, k}\left(t_{a}^{T}\right)+\theta_{p}\right] \\
& u_{p s}^{B}=\min \left[\sum_{a \in A} \delta_{a, k}\left(t_{a}^{B}\right)+\theta_{p}\right]
\end{aligned}
$$

where $u_{r p}^{c}$ is minimum travel costs by cars from origin ' $r$ ' to P\&R ' $p$ '. $u_{p s}^{c}, u_{p s}^{T}$, and $u_{p s}^{B}$ are minimum travel costs by cars, taxis, and buses from $P \& R$ ' $p$ ' to destination ' $s$ ', respectively. $\theta_{p}$ is the price of $P \& R$ ' $p$ '.

d) Modifying car travel demand based upon the minimum travel costs by combining different conditions, travel demand by cars and other modes assuming the independence of alternatives is modified using Expressions 14 to 17 ,

$$
\frac{\left(d_{w}^{c}\right)^{\text {new }}=\left(d_{w}^{c}\right)^{\text {old }}}{\exp \left(a_{c}\left(u_{r p}^{c}+u_{p s}^{c}\right)+b_{c}\right)+\sum_{m=T, B} \exp \left(a_{m}\left(u_{r p}^{c}+u_{p s}^{m}\right)+b_{m}\right)}
$$

$d_{r p}^{c}=\left(d_{w}^{c}\right)^{\text {old }}-\left(d_{w}^{c}\right)^{\text {new }}$ 


$$
\begin{aligned}
& d_{p s}^{T}=d_{r p}^{c} \cdot \frac{\exp \left(a_{T}^{\prime} u_{p s}^{T}+b_{T}^{\prime}\right)}{\exp \left(a_{T}^{\prime} u_{p s}^{T}+b_{T}^{\prime}\right)+\exp \left(a_{B}^{\prime} u_{p s}^{B}+b_{B}^{\prime}\right)} \\
& d_{p s}^{B}=d_{r p}^{C} \cdot \frac{\exp \left(a_{B}^{\prime} u_{p s}^{B}+b_{B}^{\prime}\right)}{\exp \left(a_{B}^{\prime} u_{p s}^{B}+b_{B}^{\prime}\right)+\exp \left(a_{T}^{\prime} u_{p s}^{T}+b_{T}^{\prime}\right)}
\end{aligned}
$$

where $\left(d_{w}^{c}\right)^{\text {old }}$ and $\left(d_{w}^{c}\right)^{\text {new }}$ are initial and modified travel demand by cars between OD pair ' $w$ ', respectively. $d_{r p}^{c}$ is new travel demand by private cars from origin ' $r$ ' to $\mathrm{P} \& \mathrm{R}$ ' $p$ '. $d_{p s}^{\top}$ and $d_{p s}^{B}$ are new travel demand by taxis and buses from $P \& R$ ' $p$ ' to destination ' $s$ ', respectively. $a_{j}$, $b_{j}, a_{j}^{\prime}$, and $b_{j}^{\prime}$ are constant coefficients calibrated by network data.

Step 4: Solving auto-assignment problem with fixed demand; if the demand between each OD is $d_{w}$, then the equilibrium model with fixed demand is formulated as follows [39].

$\operatorname{Min} \sum_{a \in A} \int_{0}^{v_{a}} t_{a}\left(v_{a}\right) d v$

subject to:

$$
\begin{aligned}
& \sum_{r \in R_{w}} f_{r w}=d_{w}, \quad w \in W \\
& V_{a}=\sum_{w \in W} \sum_{r \in R_{w}} f_{r w} \delta_{a r}^{w}, \quad a \in A \\
& f_{r w} \geq 0, \quad r \in R_{w}, \quad w \in W
\end{aligned}
$$

where $f_{r w}$ is the flow on route ' $r$ '. $d_{w}$ is the demand between OD pair $w \in W . v_{a}$ is the flow on link $w \in A$. $A$ is the set of links in the network. $W$ is the set of OD pairs. $R_{w}$ is the set of all routes between OD pair $w \in W . \delta_{a r}^{w}$ is one if route ' $r$ ' between OD pair $w \in W$ uses link $a \in A$, and zero otherwise.

Step 5: Updating the travel time for private cars using the BPR equation,

Step 6: Assigning taxi demand based on the updated time of private cars using auto-assignment; then, taxi volume is determined in the network.

Step 7: Adding the equivalent volume of taxis to the volume of private cars and estimating new travel time based on the BPR equation.

Step 8: Performing transit assignment using Optimal Strategies method [40],

Step 9: Estimating the volume of the bus in the links; bus demand (person) in the links is converted into bus volume (vehicle) using passenger coefficient.

Step 10: Adding the equivalent volume of bus in the links; bus volume in the links is converted into bus equivalent volume and then is added to the previous equivalent volume.

Step 11: Updating the travel time for private cars; link travel time of private cars is updated based on new equivalent passenger car using the BPR equation.

Step 12: Verifying the convergence criterion for multi-modal assignment; if Expression 22 is satisfied, proceed to step 13; otherwise, proceed to step 4.
$\sum_{a}\left|\frac{V_{a}^{n+1}-V_{a}^{n}}{V_{a}^{n}}\right| \leq \varepsilon$

where $v_{a}^{n}$ and $v_{a}^{n+1}$ are the equivalent traffic flow in link ' $a$ ' in two successive iterations.

Step 13. Verifying the convergence criterion for demand; if Expression 23 is satisfied, proceed to step 14; otherwise, proceed to step 1.

$$
\sum_{m=C, T, B}\left|\frac{\left(d_{w}^{m}\right)^{n+1}-\left(d_{w}^{m}\right)^{n}}{\left(d_{w}^{m}\right)^{n}}\right| \leq \varepsilon
$$

where $\left(d_{w}^{m}\right)^{n}$ and $\left(d_{w}^{m}\right)^{n+1}$ are demands of mode ' $m$ ' between OD pair ' $w$ ' in the two successive iterations.

Step 14: Termination of multi-modal traffic assignment; outputs of this step are traffic volumes of private cars, taxis, and buses in the links of the network.

\subsection{The upper level of the developed model considering air pollutants emission}

The upper level of cordon pricing is to maximize the social welfare function [41].

$$
\begin{aligned}
F_{1}= & \operatorname{Max} \quad S W=\operatorname{Max}\left(\sum_{w \in W} \int_{0}^{d_{w}} D_{w}^{-1}(w) d w\right. \\
& \left.-\sum_{a \in A} t_{a}^{c} \cdot v_{a}^{c}-\sum_{a \in A} t_{a}^{T} \cdot v_{a}^{T}-\sum_{a \in A} t_{a}^{B} \cdot v_{a}^{B}\right)
\end{aligned}
$$

subject to:

$0 \leq \tau \leq \tau_{\max }$

$0 \leq \theta \leq \theta_{\max }$

where $D_{w}^{-1}(w)$ is the inverse of the demand function. $d_{w}$ is total final demand of different modes for OD pair after demand convergence. $v_{a}^{C}, v_{a}^{T}$, and $v_{a}^{B}$ are flow for cars, taxis, and buses in link ' $a$ ', respectively. $t_{a}^{c}$, $t_{a}^{T}$, and $t_{a}^{B}$ are travel time for cars, taxis, and buses in link 'a', respectively. Constraints 25 and 26 refer to the maximum and minimum values of toll and price of $P \& R$, respectively.

As mentioned before, air pollutants emission may transfer from inside the cordon to its outside. Therefore, we can reduce air pollutants emission in the whole network, which actually means reducing air pollutants emission outside the cordon using the following model.

$$
\begin{aligned}
F_{1}= & \operatorname{Max} \operatorname{SW}=\operatorname{Max}\left(\sum_{w \in W} \int_{0}^{d_{w}} D_{w}^{-1}(w) d w\right. \\
& \left.-\sum_{a \in A} t_{a}^{c} \cdot v_{a}^{c}-\sum_{a \in A} t_{a}^{T} \cdot v_{a}^{T}-\sum_{a \in A} t_{a}^{B} \cdot v_{a}^{B}\right) \\
F_{2}= & \operatorname{Min}\left(E^{\text {Total }}\right)=\operatorname{Min}\left(\sum_{a \in A} E_{a}^{\text {Total }}\right)
\end{aligned}
$$

subject to:

$$
0 \leq \tau \leq \tau_{\max }
$$


$0 \leq \theta \leq \theta_{\max }$

where $E_{a}^{\text {Total }}$ and $E^{\text {Total }}$ present the total emission in link 'a' and the whole network, respectively.

Thus, the developed model guarantees that emission will decrease outside the cordon and network along with an increase in social welfare.

In this paper, three types of air pollutants are considered which include carbon monoxide (CO), carbon hydrate (HC), and nitrogen oxides (NOx). Therefore, the total emission of air pollutants in each link is as follows:

$E_{a}^{\text {Total }}=W_{1} E_{a}^{C O}+W_{2} E_{a}^{H C}+W_{3} E_{a}^{N O x}$

where $w_{1}, w_{2}$, and $w_{3}$ are the constant coefficients that indicate the importance of each of the air pollutants.

Moreover, Expression 32 is used for air pollutants emission model.

$E_{a}^{i}=a_{i}+b_{i} \bar{S}_{a}+c_{i} \bar{S}_{a}^{2}+\frac{d_{i}}{\bar{S}_{a}}$

where $E_{a}^{i}$ is the emission of pollutant ' $i$ ' in link 'a' (g/ $\mathrm{km} / \mathrm{veh}) . \overline{\mathrm{s}}_{\mathrm{a}}$ is average speed of traffic flow in link ' $a$ ' $(\mathrm{km} / \mathrm{h}) . a_{i}, b_{i}, c_{i}$, and $d_{j}$ are constant coefficients.

\section{SOLUTION ALGORITHM OF THE DEVELOPED MODEL}

\subsection{Method and solution algorithm}

Pricing problem is a non-convex optimization problem, for which it is difficult to find the optimum solution using standard optimization methods. Therefore, it is necessary to apply a global optimization method to solve the developed model. In addition, multi-objective optimization models are more complex than single-objective optimization models and different methods of solution should be applied [42].

Evolutionary algorithms (EAs) are one of the popular algorithms to solve multi-objective optimization. The first actual implementation of what is now called a multi-objective evolutionary algorithm (MOEA) is Schaffer's vector evaluation genetic algorithm (VEGA), which was introduced in the mid-1980s, mainly aimed to solve problems in machine learning [43, 44, 45]. Since then, a wide variety of algorithms has been proposed in literature [46-48].

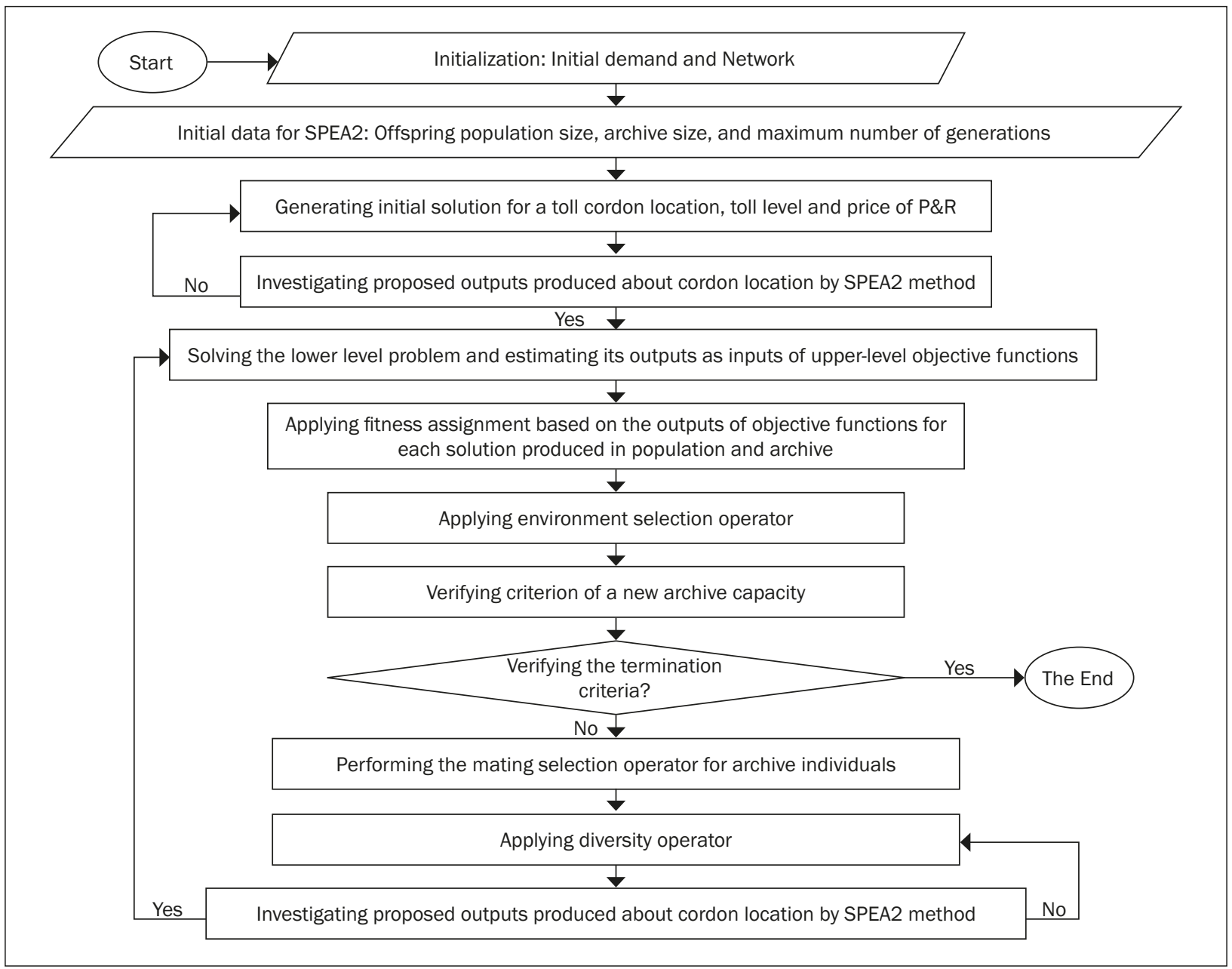

Figure 2 - Algorithm for the solution of the developed model 
SPEA2 is a member of Pareto-based approach group. SPEA algorithm was introduced by Zitzler and Thiele [49]. SPEA uses an archive-containing non-dominated solutions which were previously found. In each generation, non-dominated individuals are copied to the external non-dominated set. For each individual in this external set, a strength value is computed. This strength is similar to the ranking value of MOGA, since it is proportional to the number of solutions dominated by a certain individual. In SPEA, the fitness of each member of the current population is computed according to the strength of all external non-dominated solutions that dominate it. Additionally, a clustering technique called 'average linkage method' is used to keep diversity. But, SPEA2 approach has three main differences with respect to its predecessor [50]: (1) It incorporates a fine-grained fitness assignment strategy which takes into account the number of individuals that dominate it and the number of individuals by which it is dominated for each individual; (2) It uses the nearest neighbour density estimation technique which guides the search more efficiently, and (3) It has an enhanced archive truncation method that guarantees the preservation of boundary solutions.

According to the developed model and using SPEA2 method [42], steps of the algorithm for the solution of the developed model are shown in Figure 2.

\subsection{An innovative approach for logical constraint of SPEA2}

Dealing with logical constraint is a major issue in the application of meta-heuristic algorithms. Because these algorithms use random processes to produce solutions, the outputs generated by such algorithms may be illogical in some cases. To reject or modify SPEA2 output due to logical constraint, an innovative and reasonable method has been developed in this study. This innovative method includes two stages as follows:

\subsubsection{Specifying the cordon boundary}

According to SPEA2 outputs, the cordon boundary is determined at this stage. Assuming the specified node coordinate and the network adjacent matrix, the following steps are taken to determine the cordon boundary.

Step 1: Determining the start node; based upon the coordinate of all the selected nodes by SPEA2, the node with maximum ' $x$ ' coordinate $\left(x_{\max }\right)$ and minimum ' $y$ ' coordinate $\left(\mathrm{y}_{\min }\right)$ is determined as a starting node.

Step 2: Determining the mid-nodes; based upon the starting node and the road network adjacent matrix, the possible intermediate nodes are detected. Then, according to the angle created between the arcs connecting the starting node to potential mid-nodes and the horizon level (-x) (less than 360 degrees), the node connected to the arc with maximum angle is selected as the mid-node.

Step 3: Specifying the line equation; using the coordinate of the start node and mid-node, the line segment equation is specified.

Step 4: Verifying stopping criteria; if the mid-node is already selected in step 2, stop. Otherwise, take the mid-node as a new start point and proceed to step 2 .

Finally, the cordon boundary will be determined based on the outputs of SPEA2.

\subsubsection{Rejecting or modifying the cordon proposed by SPEA2}

In fact, the suitability of the outputs generated by SPEA2 is a response to the two following questions:

1) What is the status of the location of other unselected nodes in relation to cordon boundary (inside, outside, or on the boundary of cordon)?

2) If the unselected node locations are located outside the boundary or on the boundary, it is accepted. Otherwise, the initial outcome of the algorithm will be modified or rejected.

Therefore, to answer these questions, the following steps are taken:

Step 1: Determining the basic node; a given node inside the cordon is selected as the basic node.

Step 2: Specifying the line segment equation for the unselected node; based upon the coordinate of the nodes, the equation of the line segment connecting the basic node to the unselected node is specified.

Step 3: Calculating the total number of cross points; the total number of cross points of the line segment is connected to the unselected node and all of the line segments of cordon boundary are calculated based upon the equations of those line segments.

Step 4: Investigating the location of unselected nodes in relation to the cordon boundary; an examination of the different examples has proven that, if the number of cross points (result of step 3 ) is even, the unselected node is located inside the cordon. Otherwise, the unselected node is located outside or on the cordon boundary.

Step 5: Modifying or rejecting the proposed boundary cordon; if the number of unselected nodes inside the boundary is less than $5 \%$ of the total selected nodes, the unselected nodes inside the cordon are modified. Otherwise, the proposed boundary is rejected and new outputs should be generated by SPEA2.

\section{NUMERICAL EXAMPLE AND DISCUSSION}

In order to apply the developed model and present the discussion, the Sioux Falls network is used as a numerical example. The Sioux Falls network is shown in Figure 3. 


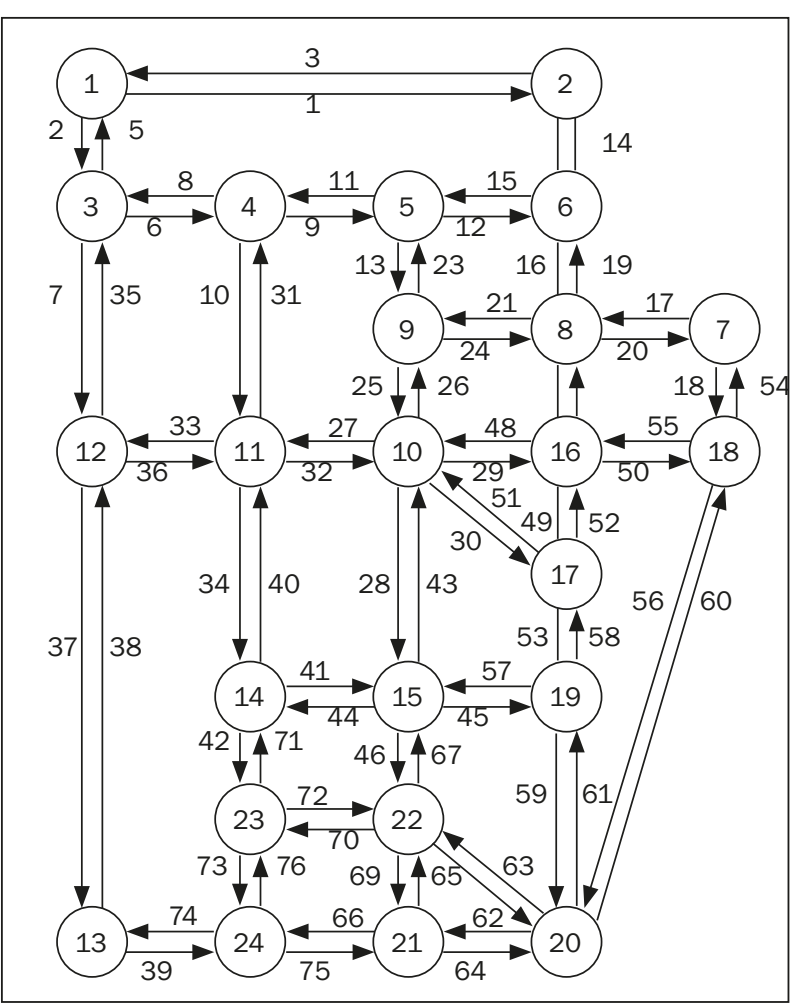

Figure 3 - Sioux Falls network

The Sioux Falls network consists of 24 nodes and 76 links. Travel cost function is as follows:

$t_{a}\left(v_{a}\right)=t_{a}^{0}\left[1.0+0.15\left[\frac{v_{a}}{C_{a}}\right]^{4}\right]$ where $t_{a}^{0}$ and $c_{a}$ are the free flow travel time and capacity of link 'a', respectively, that are given in Table 3.

In the Sioux Falls network, the four lines of bus are considered. Characteristics of bus lines service are shown in Table 4.

Travel demand of the Sioux Falls network presented in Wang et al.'s (2013) study is considered [51]. Expressions 34 to 36 are used as the utility functions of modes.

$u_{\mathrm{w}}^{c}=-0.0101 t_{\mathrm{w}}^{c}$

$u_{w}^{T}=-0.2613-0.1096 t_{w}^{T}$

$u_{w}^{B}=-0.6936-0.1257 t_{w}^{B}$

where $t_{w}^{c}, t_{w}^{T}$, and $t_{w}^{B}$ are travel time by cars, taxis, and buses between OD pair $w \in W$, respectively.

To consider drivers' behaviour change due to the existence of P\&R at the cordon boundary, Expressions 37 to 39 are used as the utility functions for shifting from cars to taxis or buses (car only, car-taxi, and carbus).

$$
\begin{aligned}
& u_{\text {car only }}=-0.0284 t_{p s}^{c} \\
& u_{\text {car taxi }}=1.21-0.0451 t_{p s}^{T} \\
& u_{\text {car bus }}=1.24-0.0432 t_{p s}^{B}
\end{aligned}
$$

where $t_{p s}^{C}, t_{p s}^{T}$, and $t_{p s}^{B}$ are travel time by cars, taxis, and buses between P\&R ' $p$ ' and destination 's', respectively.

Table 3. Free flow travel time and capacity of the network links

\begin{tabular}{|c|c|c|c|c|c|c|c|c|c|c||c||}
\hline Link & FTT & Cap. & Link & FTT & Cap. & Link & FTT & Cap. & Link & FTT & Cap. \\
\hline \hline 1 & 6 & 2,590 & 20 & 3 & 784 & 39 & 4 & 509 & 58 & 2 & 482 \\
\hline 2 & 4 & 2,340 & 21 & 10 & 505 & 40 & 4 & 488 & 59 & 4 & 500 \\
\hline 3 & 6 & 2,590 & 22 & 5 & 505 & 41 & 5 & 513 & 60 & 4 & 2,340 \\
\hline 4 & 5 & 496 & 23 & 5 & 1,000 & 42 & 4 & 492 & 61 & 4 & 500 \\
\hline 5 & 4 & 2,340 & 24 & 10 & 505 & 43 & 6 & 1,351 & 62 & 6 & 506 \\
\hline 6 & 4 & 1,711 & 25 & 3 & 1,392 & 44 & 5 & 513 & 63 & 5 & 508 \\
\hline 7 & 4 & 2,340 & 26 & 3 & 1,392 & 45 & 3 & 1,456 & 64 & 6 & 506 \\
\hline 8 & 4 & 1,711 & 27 & 5 & 1,000 & 46 & 3 & 960 & 65 & 2 & 523 \\
\hline 9 & 2 & 1,778 & 28 & 6 & 1,351 & 47 & 5 & 505 & 66 & 3 & 489 \\
\hline 10 & 6 & 491 & 29 & 4 & 485 & 48 & 4 & 485 & 67 & 3 & 960 \\
\hline 11 & 2 & 1,778 & 30 & 8 & 499 & 49 & 2 & 523 & 68 & 5 & 508 \\
\hline 12 & 4 & 495 & 31 & 6 & 491 & 50 & 3 & 1,960 & 69 & 2 & 523 \\
\hline 13 & 5 & 1,000 & 32 & 5 & 1,000 & 51 & 8 & 499 & 70 & 4 & 500 \\
\hline 14 & 5 & 496 & 33 & 6 & 491 & 52 & 2 & 523 & 71 & 4 & 492 \\
\hline 15 & 4 & 495 & 34 & 4 & 488 & 53 & 2 & 482 & 72 & 4 & 500 \\
\hline 16 & 2 & 490 & 35 & 4 & 2,340 & 54 & 2 & 2,340 & 73 & 2 & 508 \\
\hline 17 & 3 & 784 & 36 & 6 & 491 & 55 & 3 & 1,968 & 74 & 4 & 509 \\
\hline 18 & 2 & 2,340 & 37 & 3 & 2,590 & 56 & 4 & 2,340 & 75 & 3 & 489 \\
\hline 19 & 2 & 490 & 38 & 3 & 2,590 & 57 & 3 & 1,456 & 76 & 2 & 508 \\
\hline
\end{tabular}


Table 4 - Characteristics of bus lines

\begin{tabular}{||c|c|c|c|c|}
\hline Lines & Line 1 & Line 2 & Line 3 & Line 4 \\
\hline \hline Headway $(\mathrm{min})$ & 5 & 15 & 5 & 10 \\
\hline Speed $(\mathrm{Km} / \mathrm{h})$ & 20 & 15 & 25 & 15 \\
\hline Stations (nodes) & $11,10,16,17,19$ & $2,6,8,16$ & $8,9,10,15,22,21$ & $1,3,12,11,10$ \\
\hline
\end{tabular}

Table 5 - Constants value for pollutants emission including $\mathrm{CO}, \mathrm{HC}$, and NOx

\begin{tabular}{||c|c|c|c|c||}
\hline Mode & $\mathrm{a}$ & $\mathrm{b}$ & $\mathrm{c}$ \\
\hline \multicolumn{5}{|c|}{ Carbon Monoxide (CO) } \\
\hline Car & +32.58 & 0.574 & +0.004 & +310.3 \\
\hline Taxi & -46.67 & +0.708 & -0.003 & +1410 \\
\hline Bus & +19.43 & -0.330 & +0.001 & 0 \\
\hline \multicolumn{5}{|c|}{ Carbon Hydrate (HC) } \\
\hline Car & +0.901 & -0.008 & 0 & +63.68 \\
\hline Taxi & +3.153 & -0.058 & 0 & 0 \\
\hline Bus & +10.12 & -0.077 & 0 & 0 \\
\hline \multicolumn{7}{|c|}{ Nitrogen 0xides (NOx) } \\
\hline Car & +0.843 & +0.017 & 0 & +26.56 \\
\hline Taxi & +0.850 & +0.003 & -0.011 & +1383 \\
\hline Bus & -82.76 & +1.902 & & 0 \\
\hline
\end{tabular}

To determine the weight of each air pollutant, the clearance costs of a gram of them are used and the following results are obtained:

$E_{a}^{\text {Total }}=0.19 E_{a}^{c 0}+0.21 E_{a}^{H C}+0.6 E_{a}^{\text {NOx }}$

The coefficients of the air pollutants emission model are given in Table 5 for each of the air pollutants and the transportation modes.

Given the expressed assumptions, the algorithm of the developed model (bi-level multi-objective optimization model) is implemented using Matlab software. Then, two objective functions $\left(F_{1}\right.$ and $\left.F_{2}\right)$, which are social welfare and air pollutants emission, are considered simultaneously and non-dominated results (set of optimal results) are extracted based upon SPEA2 method (with the maximum number of generations: 300). The non-dominated results of the developed model are presented in Table 6.

Position of non-dominated results in the objective space or Pareto front is depicted in Figure 4.

The convex curve is formed by non-dominated results that confirms the validity of the developed model. This curve reveals that the cordon pricing scheme is a multi-objective problem, indicating that air pollutants emission will not necessarily decrease by an increase in social welfare. Therefore, the decision maker (DM) can choose each of all non-dominated results as an optimal result. This selection is exactly related to the decision maker.

Based upon the non-dominated results of the developed model with two objective functions $\left(F_{1}\right.$ and $\left.F_{2}\right)$, we have:
1) The objective function $F_{1}$ changes in the range of 930,932 to 990,523 trip-minute. The maximum value of $F_{1}$ objective function (the best situation) $(990,523$ trip-minute) is equivalent to $22,289 \mathrm{~kg}$ in the objective function $F_{2}$ (result 'A' in Figure 4).

2) The objective function $F_{2}$ changes in the range of 22,289 to $22,037 \mathrm{~kg}$. The minimum value of $F_{2}$ objective function (the best situation) $(22,037 \mathrm{~kg})$ is equivalent to 930,932 trip-minute in the objective function $F_{1}$ (result ' $B$ ' in Figure 4).

If the social welfare objective function is more important than air pollutants emission (air quality) objective function for decision makers, they can choose result ' $A$ '. If the air pollutants emission (air quality) objective function is more important than the social welfare objective function, they can select result ' $B$ '.

Moreover, according to the non-dominated results of the developed model, we can conclude: by choosing another result (changing from result ' $A$ ' to result ' $B$ ') in the objective space, we can create the best situation for air pollutants emission objective function $\left(F_{2}\right)$, while the social welfare objective function $\left(F_{1}\right)$ is only reduced to $6.02 \%$.

Results ' $A$ ' and ' $B$ ' in the solution space correspond to the specific features of cordon location, toll level, and price of $\mathrm{P} \& \mathrm{R}$, as presented in Table 7 .

Comparison of the results (changing from result ' $A$ ' to result ' $B$ ') shows that:

- Cordon area decreases (the number of the nodes in the cordon decreases);

- Toll level increases by $14.53 \%$;

- Price of P\&Rs decreases by $21.39 \%$; 
Table 6 - Non-dominated results of the developed model

\begin{tabular}{|c|c|c||}
\hline Non-dominated results & $\mathrm{F}_{1}:$ Social welfare function & $\mathrm{F}_{2}$ : Air pollutants emission function \\
\hline \hline 1 & $981,192.2$ & $22,174.4$ \\
\hline $2(\mathrm{~A})$ & $990,522.9$ & $22,288.7$ \\
\hline 3 & $971,970.6$ & $22,139.6$ \\
\hline 4 & $951,092.9$ & $22,077.4$ \\
\hline $5(\mathrm{~B})$ & $930,932.1$ & $22,037.0$ \\
\hline 6 & $949,145.9$ & $22,068.9$ \\
\hline 7 & $949,255.3$ & $22,070.3$ \\
\hline 8 & $958,199.4$ & $22,090.1$ \\
\hline
\end{tabular}

Table 7 - Features of non-dominated results ' $A$ ' and ' $B$ '

\begin{tabular}{|c|c|c|c||}
\hline Result & Nodes in the Cordon & Toll Level $(\mathrm{h})$ & Price of P\&R $(\mathrm{h})$ \\
\hline \hline A & $7,10,16,17,18$ & 3.427 & 0.187 \\
\hline B & $7,10,16,17$ & 3.925 & 0.147 \\
\hline
\end{tabular}

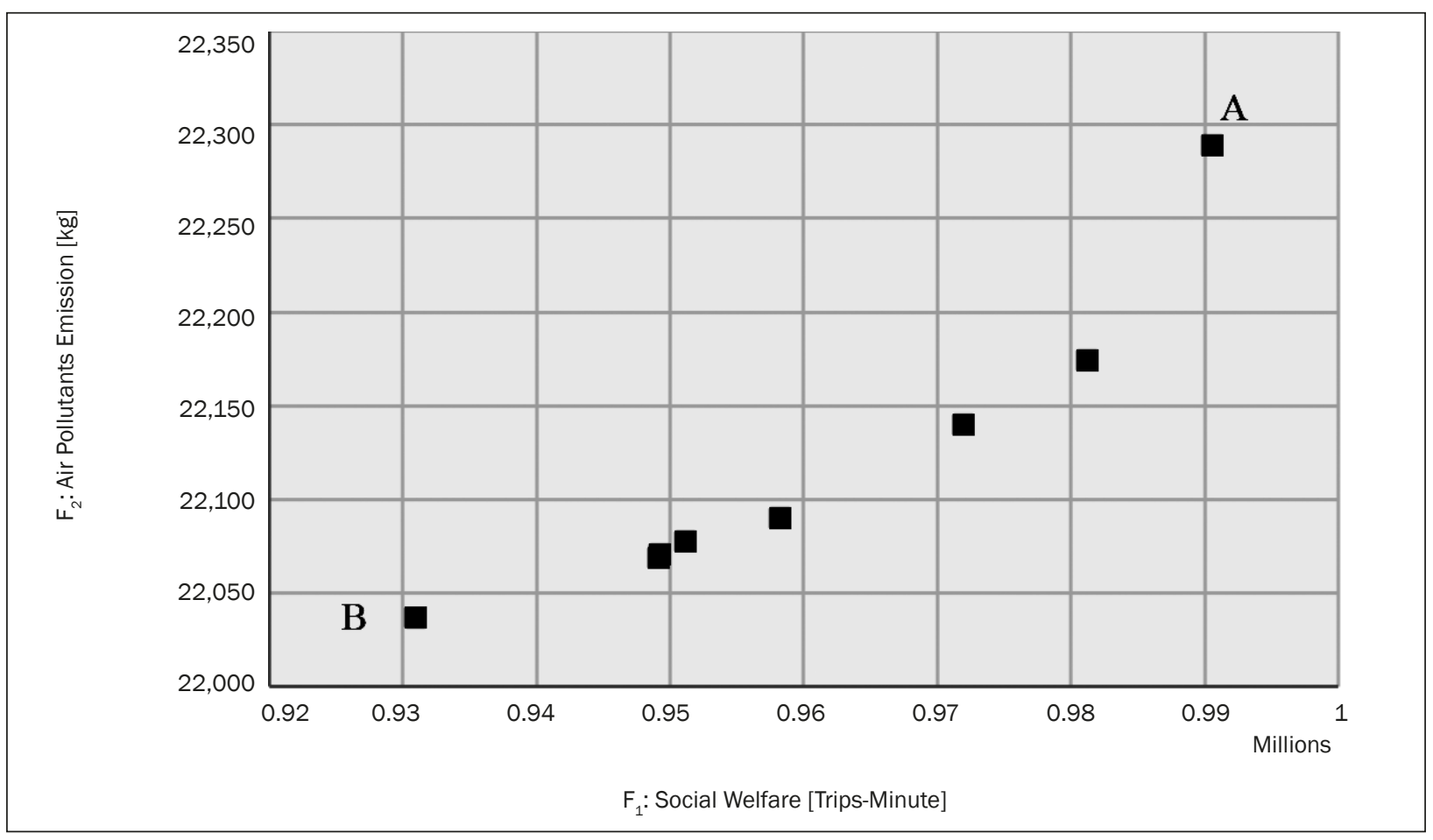

Figure 4 - Pareto front in the objective space $\left(F_{1}\right.$ and $\left.F_{2}\right)$

Therefore, air quality outside the cordon and the whole network can be improved by selecting another result (result ' $B$ '). So, by the selection of this result, we should decrease the cordon area and the price of P\&R and increase the toll level. Note that these changes are not fixed and depend on the network and demand.

\section{CONCLUSION}

This paper considered the issue of air pollutants emission (air quality) in the cordon pricing and P\&R scheme. It seems that, although the air pollutants emission decreases within the cordon by the imple- mentation of cordon pricing, it may increase outside the cordon and the whole network. In fact, due to the implementation of cordon pricing policy, air pollutants emission may transfer from inside the cordon to its outside. Therefore, cordon pricing scheme is a multi-objective problem. To consider this problem, a multi-objective bi-level optimization model was developed. Then, an algorithm was presented according to the second version of strength Pareto evolutionary algorithm (SPEA2) for solving multi-objective bi-level optimization model. Afterwards, the developed model 
was applied to the Sioux Falls network as a numerical example.

The results showed that this model can be a useful tool for the simultaneous, optimal, and sustainable determination of cordon location, toll level, and price of P\&R. Also, the results disclosed that the cordon pricing scheme is a multi-objective problem due to the formation of Pareto front in the objective space. Therefore, it is necessary to consider air pollutants emission (air quality) objective in cordon pricing and P\&R scheme. In addition, there is air pollution problem in real-world networks, which should be taken into consideration in order to increase the public acceptance of users. Moreover, the results revealed that, by choosing another non-dominated result in the solution space, we can reduce air pollutants emission (air quality) outside the cordon and the whole network without a significant reduction in social welfare.

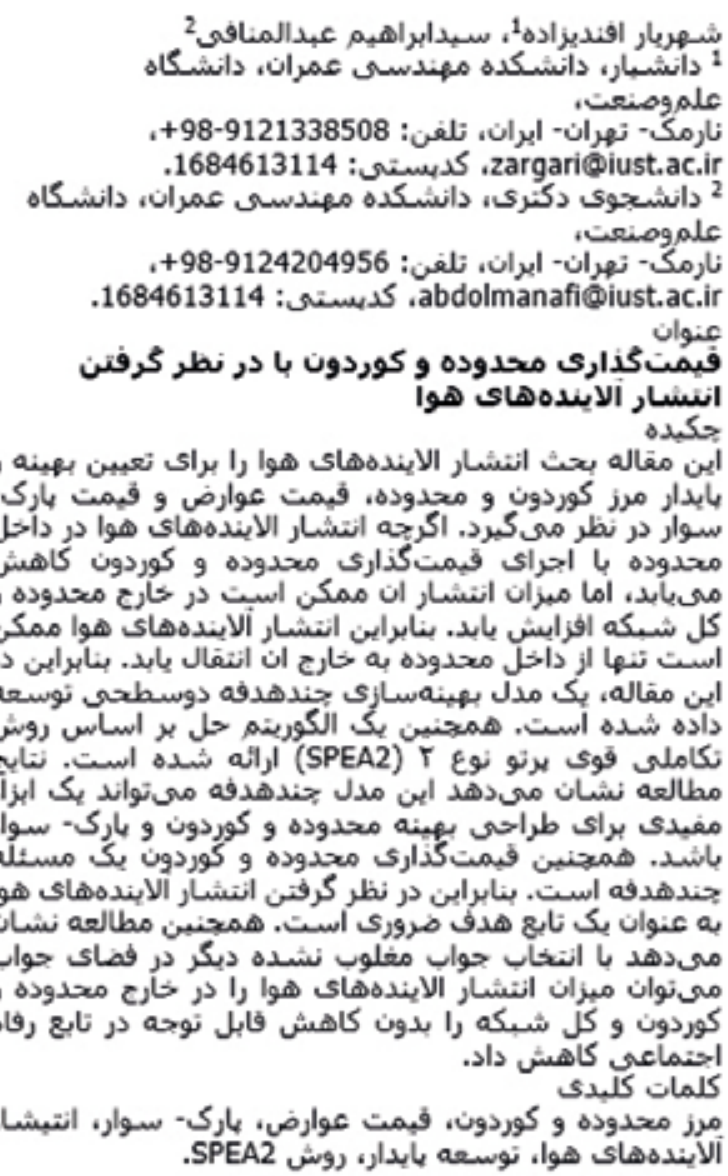

\section{REFERENCES}

[1] Wu D, Yin Y, Lawphongpanich S, Yang $H$. Design of more equitable congestion pricing and tradable credit schemes for multimodal transportation network. Transportation Research Part B. 2012;46(9):12731287.
[2] Duranton G, Turner M. The fundamental law of road congestion: evidence from US cities. American Economic Review. 2011;101(6):2016-2052; 2011.

[3] Goodwin P, Noland RB. Building new roads really does create extra traffic: a response to Prakash et al. Applied Economics. 2003;35(13):1451-1457.

[4] Lindsey CR, Verhoef ET. Traffic congestion and congestion pricing. In: Handbook of transport systems and traffic control (eds., Button KJ, Hensher DA). Elsevier Science; 2001.

[5] Levinson DM. Financing transportation networks. Edward Elgar; 2002.

[6] Santos G. Urban congestion charging - a second-best alternative. Journal of Transport Economics and Policy. 2004;38(3):345-369.

[7] Yang H, Verhoef ET. Guest editorial: road pricing problems: recent methodological advances. Networks and Spatial Economics. 2004;4(22):131-133.

[8] Verhoef ET. The economics of regulating road transport. Edward Elgar; 1996.

[9] May AD, Milne DS. Effects of alternative road pricing systems on network performance. Transportation Research Part A. 2000;34(6):407-436.

[10] May AD, Milne DS, Shepherd SP, Sumalee A. Specification of optimal cordon pricing locations and charges. Transportation Research Record. 2002;1812:60-68.

[11] Mun S, Konishi K, Yoshikawa K. Optimal cordon pricing. Journal of Urban Economics. 2003;54(1):21-38.

[12] Santos G, Newbery D, Rojey L. Static vs. demand sensitive models and the estimation of efficient cordon tolls: an exercise for eight English towns. Transportation Research Record. 2001;1747:44-50.

[13] Santos G. Double cordon tolls in urban areas to increase social welfare. Transportation Research Record. 2002;1812:49-55.

[14] Afandizadeh Sh, Yadak M, Kalantari N. Simultaneous determination of optimal toll locations and toll levels in cordon-based congestion pricing problem (case study of Mashhad city). International Journal of Civil Engineering. 2011;9(1):33-40.

[15] Verhoef ET. Second-best congestion pricing in general networks: heuristic algorithms for finding second-best optimal toll levels and toll points. Transportation Research Part B. 2002;36(8):707-729.

[16] Yang $H$, Zhang XN. Determination of optimal toll levels and toll locations of alternative congestion pricing schemes. Proceedings of the 15th International Symposium on Transportation and Traffic Theory (ed., TayIor, MAP). Pergamon; 2002, p. 519-540.

[17] Liu Z, Meng Q, Wang S. Variational inequality model for cordon-based congestion pricing under side constrained stochastic user equilibrium conditions. Transportmetrica A: Transport Science. 2014;10(8):693704.

[18] De Palma A, Lindsey R. Congestion pricing with heterogeneous travelers: A general-equilibrium welfare analysis. Networks and Spatial Economics. 2004;4(2):135160.

[19] Marcotte P, Zhu DL. Equilibria with infinitely many differentiated classes of customers. In: Complementarity and Variational Problems-State of the Art (eds., Ferris MC, Pang JS). SIAM, Philadelphia, PA; 2000. 
[20] Mayet J, Hansen M. Congestion pricing with continuously distributed values of time. Journal of Transport Economics and Policy. 2000;34(3):359-370.

[21] Nagurney A. A multiclass, multicriteria traffic network equilibrium model. Mathematical and Computer Modeling. 2000;32(3-4):393-411.

[22] Yang $\mathrm{H}$, Huang $\mathrm{HJ}$. The multiclass, multicriteria traffic network equilibrium and system optimum problem. Transportation Research Part B. 2004;38(1):1-15.

[23] Meng Q, Liu Z, Wang S. Optimal distance tolls under congestion pricing and continuously distributed value of time. Transportation Research Part E. 2012;48(5):937-957.

[24] Viegas JM. Making urban road pricing acceptable and effective: searching for quality and equity in urban mobility. Transport Policy. 2001;8(4):289-294.

[25] Paulley N. Recent studies on key issues in road pricing. Transport Policy. 2002;9(3):175-177.

[26] Raux C, Souche S. The acceptability of urban road pricing - A theoretical analysis applied to experience in Lyon. Journal of Transport Economics and Policy. 2004;38(2):191-215.

[27] Santos G, Rojey L. Distributional impacts of road pricing: the truth behind the myth. Transportation. 2004;31(1):21-42.

[28] Yang $\mathrm{H}$, Meng Q, Hau TD. Optimal integrated pricing in a bi-modal transportation network. In: Lee DH, editor. Urban and Regional Transportation Modeling: Essays in Honor of David Boyce. 2004: p. 134-156.

[29] Liu Z, Wang S, Meng Q. Optimal joint distance and time toll for cordon-based congestion pricing. Transportation Research Part B. 2014;69:81-97.

[30] Liu Z, Meng Q, Wang S. Speed-based toll design for cordon-based congestion pricing scheme. Transportation Research Part C. 2013;31:83-98.

[31] Bowen W. An analytical review of environmental justice research: what do we really know? Environmental Management. 2002;29(1):3-15.

[32] [32] Anderton DL, Anderson AB, Oakes JM, Fraser MR. Environmental equity: the demographics of dumping. Demography. 1994;31(2):229-248.

[33] Most MT, Sengupta R, Burguener MA. Spatial scale and population assignment choices in environmental justice analyses. The Professional Geographer. 2004;56(4):574-586.

[34] Mills GS, Neuhauser KS. Quantitative methods for environmental justice assessment of transportation. Risk Analysis. 2000;20(3):377-384.

[35] Chakraborty J. Evaluating the environmental justice impacts of transportation improvement projects in the US. Transportation Research Part D. 2006;11(55):315323.

[36] Clegg J, Smith M, Xiang Y, Yarrow R. Bi-level programming applied to optimizing urban transportation. Transportation Research Part B. 2001;35(1):41-70.

[37] Dempe S. Foundation of bi-level programming. Kluwer Academic Publishers, New York; 2002.
[38] Yang H, Bell MGH. Transport Bi-level Programming Problems: Recent Methodological Advances. Transportation Research Part B. 2001;35(1):1-4.

[39] Sheffi Y. Urban transportation networks. Massachusetts Institute of Technology, Prentice-Hall, Inc., Englewood Cliffs, N.J. 07632; 1985.

[40] Spiess H, Florian M. Optimal Strategies: a new assignment model for transit networks. Transportation Research Part B. 1989;23(2):83-102.

[41] Agnello RJ. Economic evaluation of highway system benefits. Transportation Research. 1977;11(5):365369.

[42] Zitzler E, Laumanns M, Bleuler S. A tutorial on evolutionary multi-objective optimization. Metaheuristics for Multiobjective Optimisation. Lecture Notes in Economics and Mathematical Systems. 2004;535:3-37.

[43] Schaffer JD. Multiple objective optimization with vector evaluated genetic algorithms. PhD thesis, Vanderbilt University, Nashville, TN; 1984.

[44] Schaffer JD. Multiple objective optimization with vector evaluated genetic algorithms. Proceedings of the First International Conference on Genetic Algorithms. Hillsdale, NJ: L. Erlbaum Associates Inc.; 1985. p. 93100.

[45] Schaffer JD, Grefenstette JJ. Multi-objective learning via genetic algorithms. Proceedings of the 9th International Joint Conference on Artificial Intelligence (IJCAI-85). Los Angeles, CA; 1985. p. 593-595.

[46] Coello Coello CA. A comprehensive survey of evolutionary-based multi-objective optimization techniques. Knowledge and Information Systems. 1999;1(3):269308.

[47] Coello Coello CA, Van Veldhuizen DA, Lamont GB. Evolutionary algorithms for solving multi-objective problems. New York: Kluwer Academic Publishers; 2002.

[48] Coello Coello CA, Mariano Romero CE. Evolutionary algorithms and multiple objective optimization. In: Ehrgott M, Gandibleux X, editors. Multiple Criteria Optimization: State of the Art Annotated Bibliographic Surveys. Boston: Kluwer Academic Publishers; 2002.

[49] Zitzler E, Thiele L. Multi-objective evolutionary algorithms: a comparative case study and the strength Pareto approach. IEEE Transactions on Evolutionary Computation. 1999;3(4):257-271.

[50] Zitzler E, Laumanns M, Thiele L. SPEA2: Improving the strength Pareto evolutionary algorithm. Technical Report 103. Zurich: Computer Engineering and Networks Laboratory (TIK); 2001.

[51] Wang S, Meng Q, Yang H. Global optimization methods for the discrete network design problem. Transportation Research Part B. 2013;50:42-60. 